\title{
Location-Based Augmented Reality Information for Bus Route Planning System
}

\author{
Komang Candra Brata ${ }^{1,2}$, Deron Liang ${ }^{2}$, Sholeh Hadi Pramono ${ }^{1}$ \\ ${ }^{1}$ Department of Computer Science and Information Engineering, National Central University, Taiwan \\ ${ }^{2}$ Departement of Electrical Engineering, University of Brawijaya, Malang, Indonesia
}

\begin{tabular}{l}
\hline \hline Article Info \\
\hline Article history: \\
Received Oct 4, 2014 \\
Revised Nov 21, 2014 \\
Accepted Dec 10, 2014
\end{tabular}

\section{Keyword:}

Augmented Reality

Bus Route Planning

Mobile Application

Navigation

\begin{abstract}
Bus Route Planner applications will unfold their full potential when bus passengers are enabled to get information about the shortest path route, make a travel plan and get the correct buses in order to reduce the travel time. However, all these information are provided in text based and map view. It is difficult to understand them for the person who does not know place in the map. This paper describes the android base application of Augmented Reality (AR) that has feature to support the action of a bus user in an innovative and dynamic ways by putting additional information layer on smart phone camera screen and give the instruction assistant that leading the user way to the nearest bus stop. The experimental results show that, the overall functional of proposed application can be run well in various type of Android smart phone. When compared with similar bus traveling applications, the proposed application works more efficient.
\end{abstract}

Copyright $@ 2015$ Institute of Advanced Engineering and Science. All rights reserved.

\section{Corresponding Author:}

Komang Candra Brata,

Department of Computer Science and Information Engineering,

National Central University,

No. 300, Jhongda Rd., Jhongli City, Taoyuan County 32001, Taiwan, ROC.

Department of Electrical Engineering,

University of Brawijaya,

J1. Veteran, Malang 65145, East Java, Indonesia.

Email: komang.candra.brata@gmail.com

\section{INTRODUCTION}

A bus passenger dislikes uncertainty in his traveling. If he could get instant, personal, and real-time bus information traffic or solutions that help him make decision to reduce the travel time to his destination, he would have much less uncertainty to deal with.

Previous work on bus route information had proposed a range of features for route planning and direction problems. Such features include using multimodal integrated data that provided by government [1][2] and participatory sensing technique [3]. We also already developed "Taipei BusRoutePlanner", an android application that can be used to make a traveling plan and get the correct buses by provide information about bus route and bus arrival time [4]. However, all these information are provided in text based map view. This application do not provide additional information to make sure the user can go to the correct nearest bus stop or route path that application suggest, so it is difficult to understand them for the person who does not know the place and can cause the user confuse and fail to find the destination especially for foreigner. According to Taipei data, $65 \%$ of foreign visitors go to the service centers for seeking the directions to their destinations [5].

Recent improvements in the capabilities of smart phones are making the location-based augmented reality services a reality. AR is a powerful user interface technology that augments the user's real life environment with computer generated data. The technology of AR is studied in a variety of areas for over 10 
years [6]. The interest of those studies is motivated by a wide range of applications that produced, such as entertainment [7], social media [8], geographic information system (GIS) [9] and navigation [10]. The location-based augmented reality view provides a direct view of reality enhanced with additional computergenerated data, as opposed to a conventional map, which provides an abstract view from above.

In this paper, we have proposed a robust, lightweight and computationally inexpensive Augmented Reality system that can support the information that user get. This system name is "BusAR", the AR feature that provides information such as objects of bus stops and detail information that are overlaid on a smart phones camera view. BusAR is obviously contributing to improve the usability of public bus transportation and that will be a big help for a bus passenger who are not good at reading maps to understand a location of the nearest bus stop to destination.

\section{RESEARCH METHOD}

There are many methods available for marker detection and tracking in Augmented Reality, Taketomi et al [11] describe a robust outdoor tracking method that uses two stages. In the initial off line stage, a landmark database is constructed from structure from motion data and laser range finder information. After this database is constructed, landmark tracking can be used in a non-line stage to provide wide-area outdoor tracking for mobile AR applications. Stephen DiVerdi uses a vision-based approach for orientation tracking [12]. It tracks the camera orientation in real time and simultaneously creates an environment map by calculating the optical flow between successive frames. These measurements are refined with more computationally expensive landmark tracking to avoid the drift that frame-to-frame feature matching introduces. But this approach can't run on phones due to high computational costs, because the method requires extensive GPU. Sensor based tracking obtain geo-location information by the fusion of several different sensors like GPS, magnetometer and linear accelerometers to determine where annotations should appear in the camera view. AR also needs an internet connection to receive data for the information layer.

Current commercial solutions for AR browser on smart phone that use sensor based tracking approach are Wikitude [13] and Layar [14]. The sensor based approach is used to this project, because it is focused on the device's location and orientation. This means if user are in a particular place and pointing the device in a particular direction, it shows only the virtual bus stop annotations that corresponding to that place and direction that matched with user radius distance and bus stop coordinate on the database

Location-Based AR provides a direct view of reality enhanced with additional computer generated data, as opposed to a conventional map, which provides an abstract view from above. The core function and also the common goal of BusAR is simply to display mostly textual computer generated data annotations that are registered in database to anchor points such as bus stop places or objects in the real world. The first thing in every AR app is the camera to capture the real time live environment, Orientation Sensor (magnetic field sensor and accelerometer sensor), Gyroscope (Rotation detection), Global Positioning System (GPS).

\subsection{Requirements Analysis}

To extract the information which could be useful for a user, we performed a survey based on [15] to get some feedbacks and user experiences about our prototype. The following information requirements are needed for BusAR application :

1. Location-Based Augmented Reality View

Map View route planning give user information about all bus stops that available in one particular route. If the user is foreigner or new people that doesn't know the area and compass direction, he can't find the bus stop location that show on the map and may loses his way to the bus stop or go to the wrong bus stop. A big help for a user who is walking to the bus stop would be to show him which bus stops are close to him and how far the distance from his position in an intuitive way. To develop augmented reality feature, we need data from several sources. As part of this experiment scope we only dealt with information from Taipei Bus Company API and Google API. This application must provide the bus stops as augmented reality entities called Point Of Interest (POI) that could be displayed as well as details of these entities like the details of the bus stop name, distance from user, route name, upcoming bus name and type of bus (low chases or normal) and also estimate time when bus will arrive in specific bus stop. The POI's shape should be transparent so that the camera view is not completely covered. The POI must respond user touch event with show the detail information about corresponding POI. In addition, POI also update their data in real time while all possible user movements.

2. Guidance Service

There are many bus stops that close to user position, so user need to know the information about the nearest bus stop and the direction to go there. BusAR should be swapped for information about the 
direction and step by step hint to nearest bus stop that inform user when to turn left or turn right without change his application screen.

3. Navigation Service Integration

In addition to enriched the guidance service, there could be another perspective, which is activated when users choose which direction that they want to follow in instruction list view and then pointing the camera of the smartphone to the ground. A compass-based arrow could be shown in this view. According to the device's orientation it always points to the user direction waypoint. Show basic description of this waypoint, as well as the distance of it, so user is able to get the most important information to reach the next waypoint with just a quick look.

\subsection{System Architecture}

This system use 3-tier architecture for data transactions. It involves three key concepts: use web service as data service, modularity in software development, for superior performance and portablility, it separate the components of application servers that containing business logic functions and database servers that containing databases physically into different tiers/layers. There are thousands of route in Taipei and each route have many bus stop objects, this application only generate the augmented reality POI objects in one route that chosen by user. System architecture and data flow of this application are shown in Figure 1.

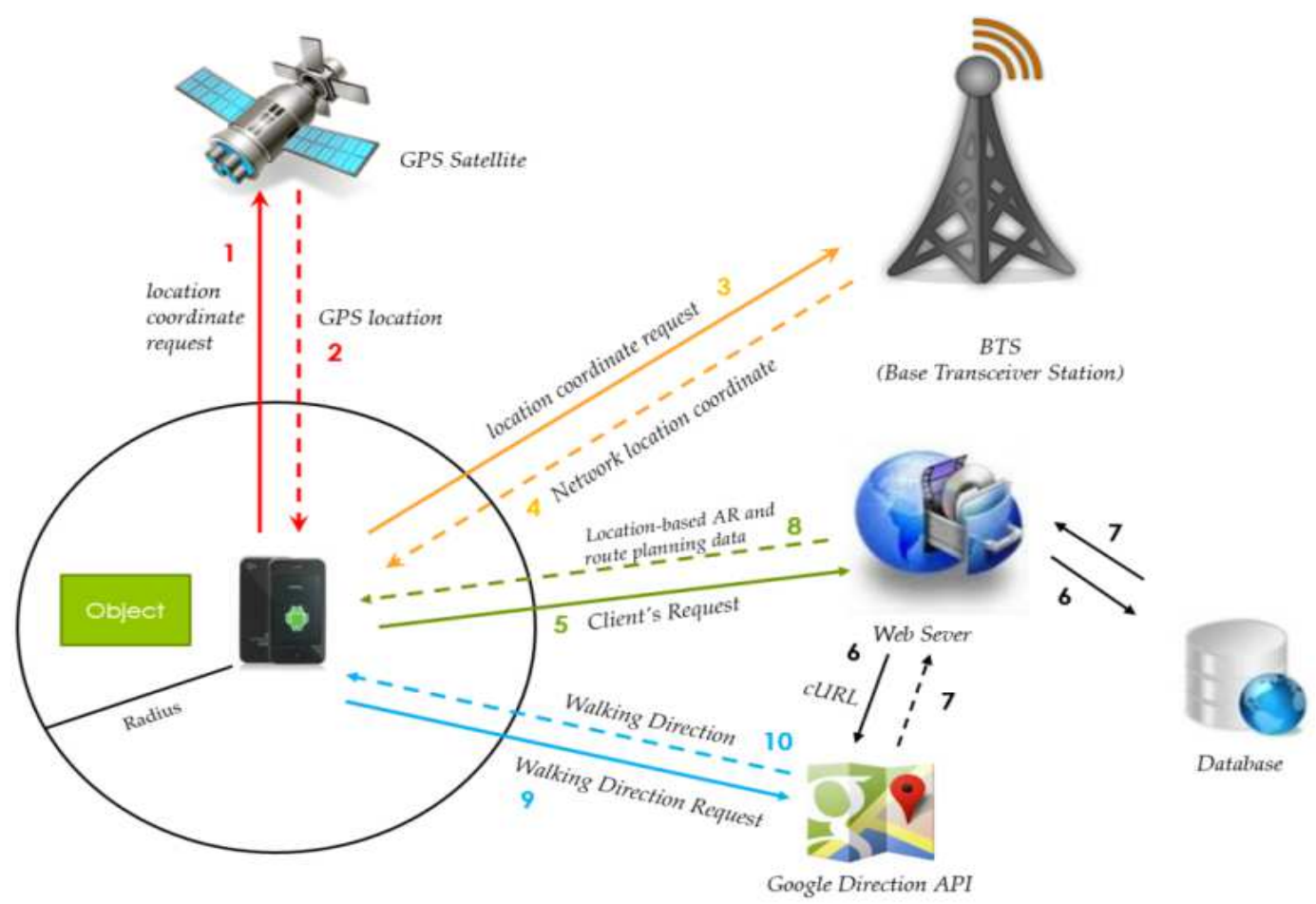

Figure 1. System architecture of BusAR environtments

Data sources are from our database that reference to Taipei Bus API and Google Direction API that provide the direction data. In order to make this system computationally fast, web services are used as part of this core system. Response that provided by the web service then decoded by client applications into an object that will be further processed in the android client application to render an augmented POI marker.

\subsection{Marker Extraction}

In order to display a POI marker to user accurately, this application need to convert the raw data from database to POI data format then generate the object marker based on user smart phone orientation. Web service is used as a bridge between clients and server. Michael C. Daconta [16] define web services are software applications that can be discovered, described, and accessed based on XML and standard Web protocols over intranets, extranets, and the Internet usually called Application Programming Interface (API). This system use JSON (JavaScript Object Notation) as the data transfer format because that JSON is faster and uses fewer resources than XML [17]. JSON is a lightweight data-interchange language, text based, easy 
to read, and have high compatibility with many popular programming languages such as $\mathrm{C}, \mathrm{C}++, \mathrm{C \#}, \mathrm{Java}$, JavaScript, Perl and Python [18]. This method works on the following principles: the process begins with collect current smart phone location and orientation using smart phone sensors then perform data request. Web service is used to process the client request and determine the data source. Next, response data from web service will be computed and transformed to augmented reality marker in client side. Finally, the generated marker will be displayed based on smart phone sensors value. The complete process flow is shown in Figure 2.

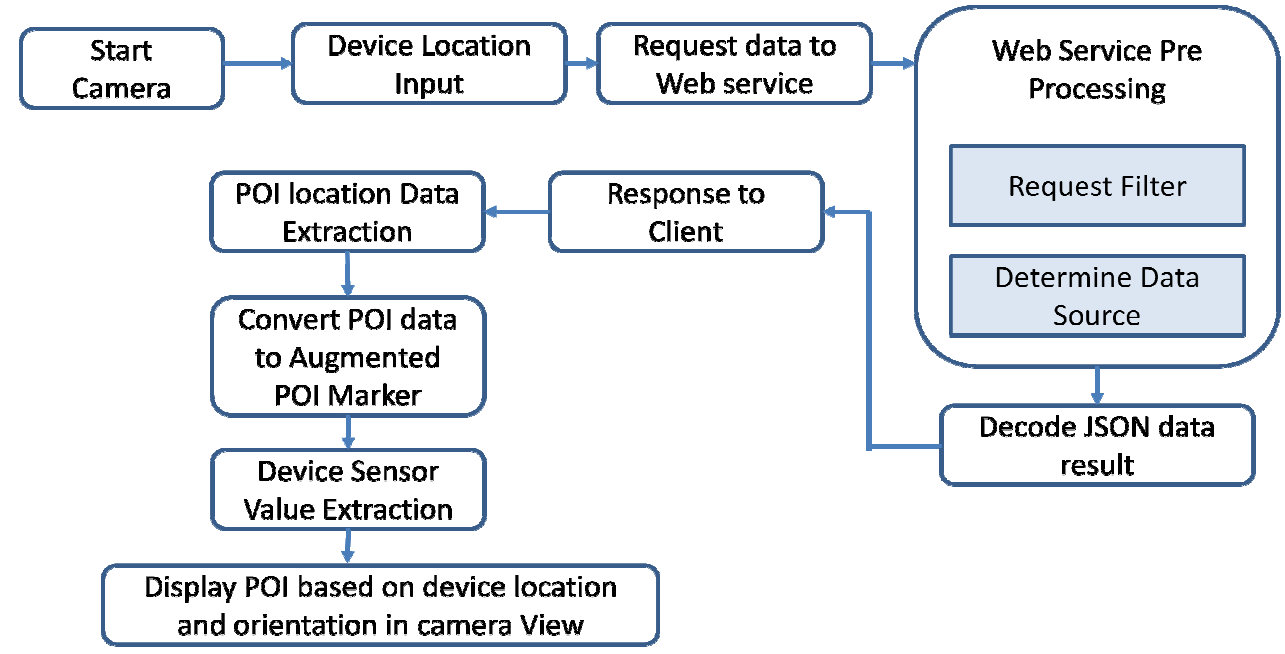

Figure 2. Process flow of BusAR system

\subsection{Marker Detection}

When the application running, augmented reality POI objects of bus stops are overlaid on camera screen. The marker detection process use the method as shown in Figure 3. This AR application send request to server and then generate the POI based on data in database. After the application get response data and phone location, it syncronize the POI with smart phone orientation and movement sensors data. So the POI marker can be displayed accurately in smartphone screen that overlaid the view of real world object.

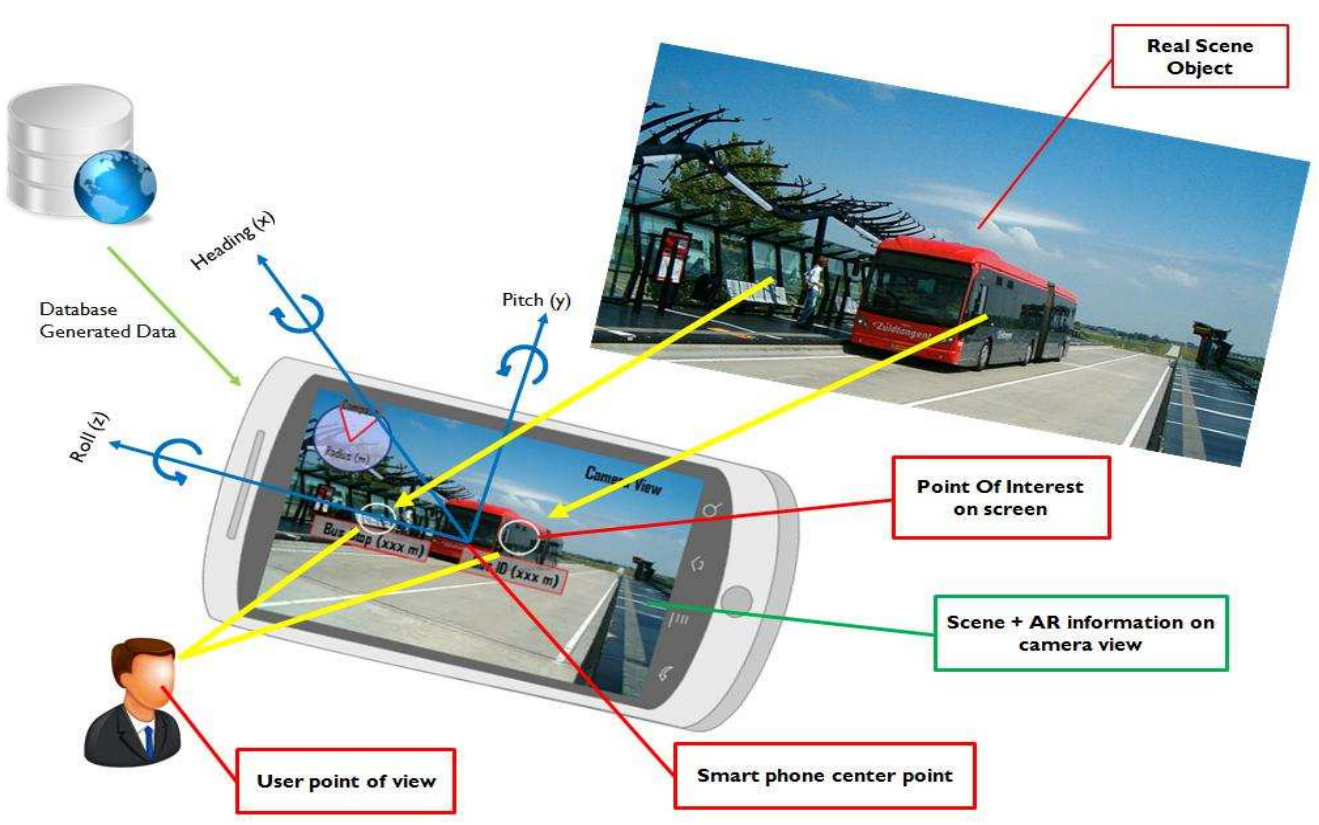

Figure 3. Marker detection process 


\subsection{Calculate the Distance}

This application have used the Great Circle Haversine Formula to determine the distance between POI marker and user location. Great-circle distance is a method known from spherical geometry to calculate the distance between two points on a curved surface like the earth. The distance is not measured based on a line through the sphere, but related to the surface of the sphere. On computer systems with low floating-point precision, Haversine formula is numerically better-conditioned for small distances [19].

$$
\begin{aligned}
& \theta=2 \arcsin \left(\sqrt{\sin ^{2}\left(\frac{\Delta \phi}{2}\right)+\cos \phi_{A} \cos \phi_{B} \sin ^{2}\left(\frac{\Delta \lambda}{2}\right)}\right) \\
& D=\theta * 6371 \mathrm{~km}
\end{aligned}
$$

Here, A corresponds to the position of the user and B to the one of the POI. Further, $\phi$ denotes the latitude and $\lambda$ the longitude of one of these positions. Finally, $\Delta \phi=\phi B-\phi A$ corresponds to the difference between two latitudes and $\Delta \lambda=\lambda \mathrm{B}-\lambda \mathrm{A}$ corresponds to the difference between two longitudes. Parameters $\phi$, $\lambda, \Delta \phi$, and $\Delta \lambda$ must be available in radians to calculate the angle $\theta$ between these two points. To determine distance $\mathrm{D}, \theta$ must be multiplied by the radius of the earth $6371 \mathrm{~km}$.

\section{RESULTS AND DISCUSSION}

As described in IEEE standards [20] [21], validation needed to check whether all software functionalities have satisfied the requirements. To evaluate the feasibility of our approach, we implemented several case studies on top of android platform. In the following, we introduce several functions of BusAR like searching nearest bus stop, inform a detail bus stop information, and guidance assistance to demonstrate the usability of the application.

\subsection{Searching Nearest Bus Stop}

MapView function allow the user to see his current location and location of available bus stops in particular bus route that chosen by user. This feature practically provides an abstract view from above. Figure 4 shows the detail map view of bus stops function. User can use this map function to see available bus stops in particular bus route that chosen by user.

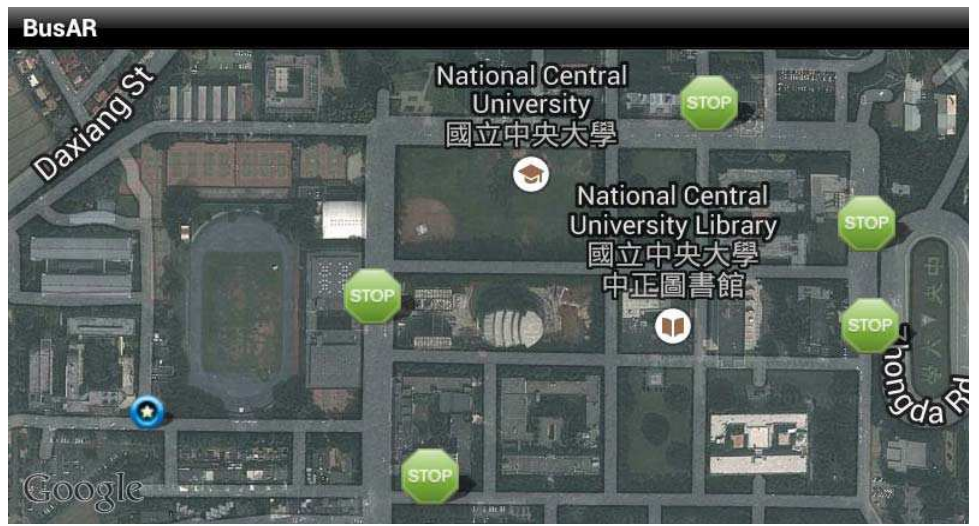

Figure 4. MapView function of BusAR application

Location information from this map-based view function can be used to create an augmented reality function that supports the user to understand the map information by show the direction and step by step hint as POI markers. This application generates the augmented POI of bus stops annotation based on user position and direction. It also calculates the distance between user current location and POIs location. Figure 5 shows that POI can guide user to the nearest bus stop by inform him when to turn left or turn right without change his application screen. 


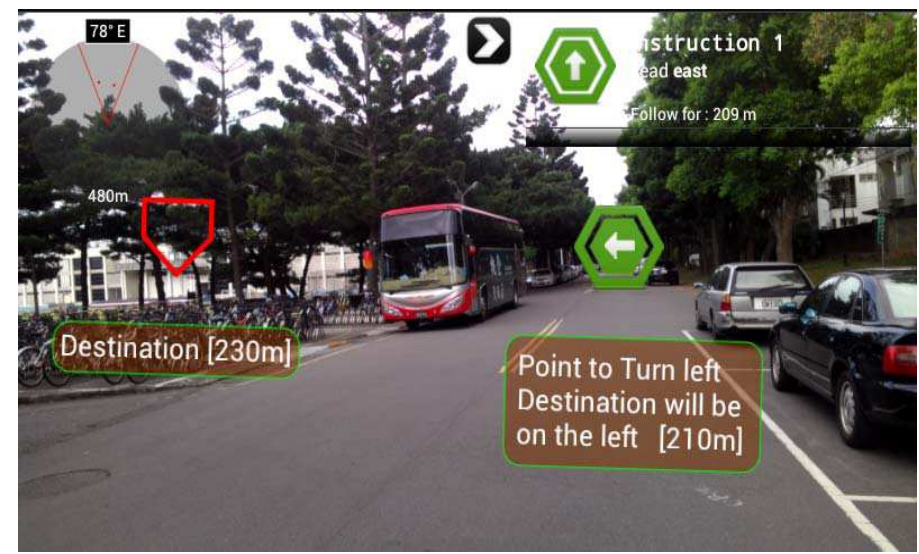

Figure 5. Augmented reality view of BusAR application

\subsection{Guidance Assistant and Bus Stop Information}

BusAR enables users to intuitively find the nearest bus stop. Additional information for user current routing instruction segment are displayed on top right corner ListView. User can choose one next waypoint to his nearest bus stop. In addition, BusAR navigation service activates a compass-based arrow that always points to the next waypoint when user pointing the camera of the smartphone to the ground. The left side of Figure 6 shows the detail view of this feature. The navigation services are propagated in real time manner based on user location to ensure a user can arrive in correct bus stop.

Using the Taipei Bus API integration service, all bus data in Taipei can be shared to all of this application users. The user can get the detail information while on travel to bus stop. The right side of Figure 6 shows the detail bus stop information function when user touch a bus stop POI marker on the screen, like bus stop name, route name, upcoming bus name and type of bus (low chases or normal) and also estimate time when bus will arrive in specific bus stop.
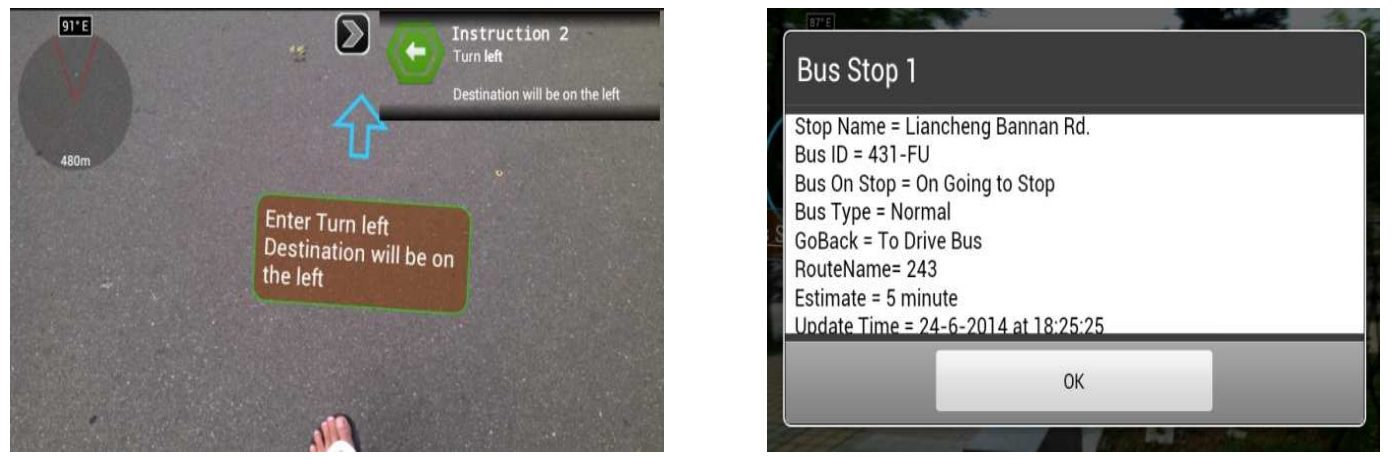

Figure 6. Augmented navigation function (left) and detail information inside POI (right)

\subsection{Result of Performance Testing}

The quality and the efficacy of BusAR application was comparatively evaluated using data usage parameter. Proposed architecture and software development model in this paper works quite well as compared to the other similar applications. Figure 5 and 6 show that our application is more user friendly as compared to earlier systems (Fun Travel in Taipei [1] and BusRotePlaner [4]). It can help user to intuitively find the nearest bus stop according their location.

In IEEE standard validation [21], data usage mentioned as one of indicator for efficiency and correctness, so we use data communication usage as efficiency indicator in comparison with other projects. To calculate data usage, this application have been run in 5 rounds with time durations are $5 \mathrm{~min}, 10 \mathrm{~min}, 15$ $\mathrm{min}, 20 \mathrm{~min}$, and $25 \mathrm{~min}$ in each round. The raw experiment data then transformed to result data by categorize them by duration time and then take the average value on each category. Figure 7 shows the peformance of proposed application, this application is much better in terms of data usage as compared to the

Location-Based Augmented Reality Information for Bus Route Planning System(Komang Candra Brata) 
earlier systems. This because the proposed system was implemented using RESTful web service technology with JSON as data exchange format, so different platforms clients can access information in an easy way via lightweight web API. Data usage in bandwidth metric at various applications and the overall performance are summed up in Tables 1.

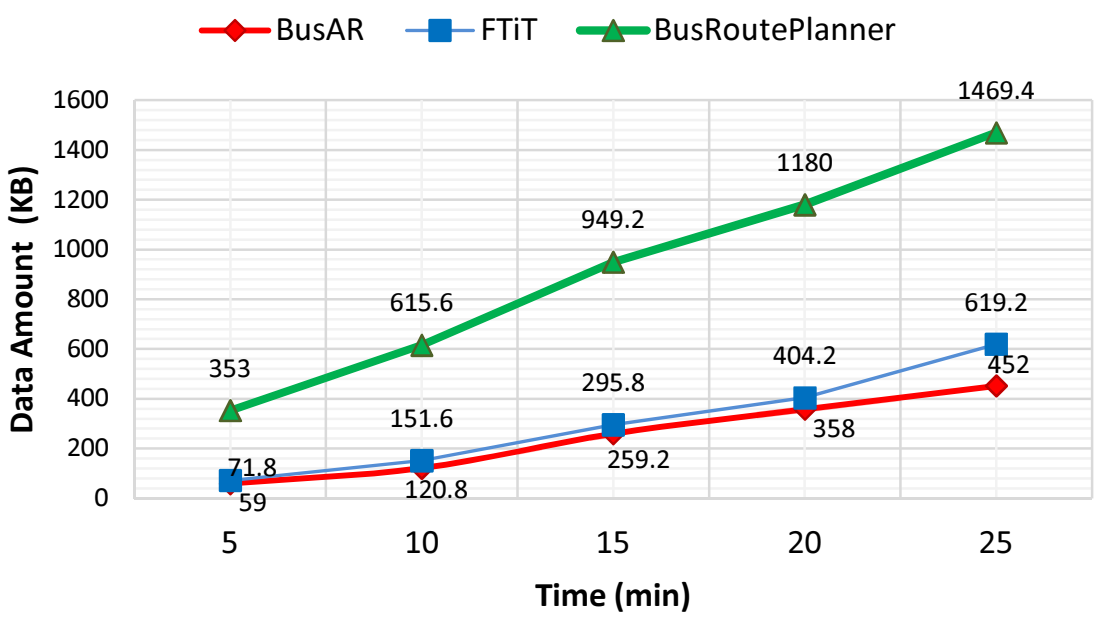

Figure 7. Data usage comparison in several times

Table 1. Average value in bandwidth metric

\begin{tabular}{ccc}
\hline BusAR (KBps) & FTiF (KBps) & BusRoutePlanner(KBps) \\
\hline 0.25 & 0.30 & 1.04 \\
\hline
\end{tabular}

\section{CONCLUSION}

This paper proposes an augmented reality application for public transportation. A bus passenger can understand the exact location of the bus stop in real world intuitively with this application. In addition, the passenger can see all bus stop information in one route such as route name, stop name, incoming bus type, arrival time and fare when select one of POI marker in the screen. This system have been tested in an uncontrolled environment and a real time environment as well. The results show that this system is more robust when compared to the other similar systems. Its real time augmented reality application so makes it improve the usability of bus transportation. It is a lightweight application that takes $0.25 \mathrm{KBps}$ data usage in average to operate.

\section{ACKNOWLEDGEMENTS}

The authors would like to thanks Taipei Goverment for give the bus company transportation data, Adam Hendra Brata for provide automatic data collector and also reviewers for their valuable comments.

\section{REFERENCES}

[1] Department of Transportation, "Fun Travel in Taipei (FTiT)", ANNUAL REPORT Taipei City Government, pp. 3031, 2012.

[2] Zhou, Chonghua; Weng, Zhiyong; Chen Xu and Zhizhe Su, "Integrated Traffic Information Service System for Public Travel Based on Smart Phones Applications: A Case in China”, I.J. Intelligent Systems and Applications, vol. 12, pp. 72-80, 2013.

[3] Zhou, Pengfei; Yuanqing Zheng; Mo Li, "How Long to Wait?: Predicting Bus Arrival Time with Mobile Phone based Participatory Sensing", ACM Journal , vol. 978-1-4503-1301-8/12/06, pp. 379-392, 2012.

[4] Software Methodology Laboratory (SML), "Bus Route Planner System", National Central University Private communication, Taiwan, 2013.

[5] Mo, Yan-chih, "Taipei introduces direction stickers for foreign tourists", Taipei Times http://www.taipeitimes.com/News/taiwan/archives/2013/04/19/2003560119 [Accessed October 9th, 2013].

[6] Ronald T. Azuma, "A Survey of Augmented Reality", Presence Tele operators and Virtual Environment, pp. 355385, 1997. 
[7] Blum Lisa, Richard Wetzel, Rod McCall, Leif Oppermann, Wolfgang Broll, “The Final TimeWarp: Using Form and Content to Support Player Experience and Presence when Designing Location-Aware Mobile Augmented Reality Games", ACM; 978-1-4503- 1210-3/12/06, pp.711-720, 2012.

[8] Balduini Marco, et al., "BOTTARI: An augmented reality mobile application to deliver personalized and locationbased recommendations by continuous analysis of social media streams", Elsevier Web Semantics: Science, Services and Agents on the World Wide Web, vol. 16, pp. 33-41, 2012.

[9] Nicholls, Gareth, "Using augmented reality as an extension to utility GIS", IST Powertech journal, pp.62-64, 2013.

[10] Wolfgang Narzt, et al., "Pervasive Information Acquisition for Mobile AR-Navigation Systems", Proceedings of the Fifth IEEE Workshop on Mobile Computing Systems \& Applications, pp.1-8, 2003.

[11] Taketomi, Takafumi; TomokazuSato; Naokazu Yokoya, "Real-time and accurate extrinsic camera parameter estimation using feature landmark database for augmented reality", Elsevier Computers \& Graphics, Vol. 35, pp. 768-777, 2011

[12] DiVerdi, Stephen; Withert, Jason; and Hollerert, Tobias, "Envisor: Online Environment Map Construction for Mixed Reality", IEEE Virtual Reality, 978-1-4244-1971-5/08, pp. 19-26, 2008.

[13] Wikitude, "Wikitude Application", 2013, http://www.wikitude.com/ [Accessed October 26th 2013].

[14] McFerran, Damien, "Layar Reality Browser review", 2013, http://www.smartphonetracker.co.uk/software-appreviews/androidos/androidnavigation/342270/layar_reality_browser_review.html [Accessed October 26th 2013].

[15] Olsson, T., \& Salo, M, "Online user survey on current mobile augmented reality applications", Mixed and Augmented Reality (ISMAR), 10th IEEE International Symposium, pp. 75-84, 2011.

[16] Daconta, M.C.; Obrst L.J.; and Smith, K.T, "The Semantic Web: A Guide to the Future of XML, Web Services, and Knowledge Management”. John Willey, 2005.

[17] Nurseitov, Nurzhan, et al. "Comparison of JSON and XML Data Interchange Formats: A Case Study." Caine 2009, pp. 157-162, 2009.

[18] Json.org,'Introducing JSON", 2013 http://json.org [Accessed December 25th 2013].

[19] Sinnott, R.W, "Virtues of the Haversine". Sky and telescope, vol. 68:2, pp. 158, 1984.

[20] IEEE Computer Society, "IEEE Standard for Software and System Test Documentation". IEEE Std 829TM - 2008, 2008.

[21] IEEE Computer Society, "IEEE Standard for System and Software Verification and Validation". IEEE Std 1012 TM2012,2012

\section{BIOGRAPHIES OF AUTHORS}

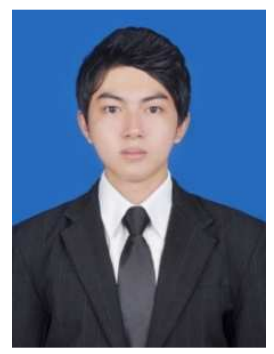

Komang Candra Brata received the MS degree in Department of Computer Science and Information Engineering, National Central University, Taiwan in 2014 as an International Dual Degree Master student between University of Brawijaya, Indonesia and National Central University, Taiwan. He completed his Bachelor degree in Department of Informatics Engineering, University of Brawijaya, Indonesia in 2012. His research interest area in the areas of software engineering and information technology, distributed systems, and augmented reality.

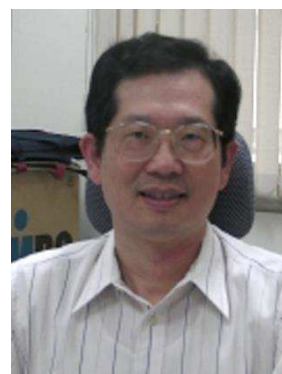

Deron Liang is a Professor in the Department of Computer Science and Information Engineering, National Central University, Taiwan. He received a BS degree in electrical engineering from National Taiwan University in 1983, an MS and a PhD in computer science from the University of Maryland at College Park, USA in 1991 and 1992 respectively. He also holds joint appointment with the Institute of Information Science (IIS), Academia Sinica, Taipei, Taiwan, Republic of China. He was with IIS from 1993 till 2001. Dr. Liang's current research interests are in the areas of software fault-tolerance, system security, distributed systems, object oriented and system reliability analysis. Dr. Liang is a member of ACM and IEEE.

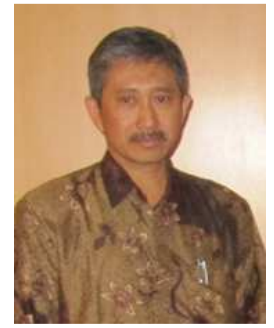

Sholeh Hadi Pramono is an senior lecturer in Department of Electrical Engineering, University of Brawijaya, Indonesia. He got Doctor degree from University of Indonesia, Indonesia. He presently work in Telecommunication Laboratory, University of Brawijaya, Indonesia as an optical telecommunication specialist. His research interest area in the areas of optical telecommunication, technology of antenna, distributed systems, and telecommunication architecture. 